\title{
Impaired consciousness and decreased glucose concentration of CSF as prognostic factors in immunocompetent patients with cryptococcal meningitis
}

\author{
Chen Zhang, Zheren Tan and Fafa Tian *id
}

\begin{abstract}
Background: Cryptococcal meningitis (CM) is the most common fungal infection of the central nervous system and has high morbidity and mortality. Almost studies about prognostic factors have largely focused on the immunocompromised population rather than immunocompetent patients. So that we sought to conduct a retrospective study to determine prognostic factors which predict the outcomes in immunocompetent patients with CM.

Methods: We retrospectively collected and analyzed the demographic and clinical data of 76 apparently immunocompetent patients with cryptococcal meningitis from January 2003 to June 2019 in China. The clinical outcome was graded by the Glasgow outcome scale (GOS) at discharge, and patients were divided into good (score of 5) and unfavorable (score of 1-4) outcome groups, potential prognostic factors were analyzed.

Results: Non-parametric test confirmed that unfavorable outcome was associated with lower glucose level of $\operatorname{CSF}(P=0.001)$, and Pearson's $\times 2$ analysis confirmed that unfavorable outcome was associated with opening pressure of $\operatorname{CSF}(>300 \mathrm{mmH} 20, P=0.038)$, impaired consciousness $(P=0.001)$, hydrocephalus $(P=0.045)$, and Shunt surgery $(P=0.045)$, and then multiple logistic regression analysis confirmed that impaired consciousness $(P=0.015)$ and lower glucose concentration of $\operatorname{CSF}(P=0.012)$ increased the likelihood of unfavorable outcome in CM patients.

Conclusion: Impaired consciousness and decreased glucose concentration of CSF were independently prognostic factors which predict the unsatisfactory outcome in immunocompetent patients with CM.
\end{abstract}

Keywords: Cryptococcal meningitis, Clinical features, Prognostic factors, HIV negative, Immunocompetent patients

\section{Background}

Cryptococcal meningitis $(\mathrm{CM})$ is the most common type of chronic infectious meningitis caused by Cryptococcus neoformans [1, 2], the reservoirs of them are mainly pigeon or other bird droppings [3]. The delay in diagnosis and treatment result in a high morbidity and a mortality rate [4-6]. majority of cryptococcosis have occurred in HIV-positive patients, but it is also been found in cases with comorbidities that result in immunosuppression, such as hematological malignancies, solid-organ transplant recipients, and in patients on

\footnotetext{
* Correspondence: xysntff@sina.com; xysnzhangchen@126.com Department of Neurology, Xiangya Hospital, Central South University, 87 Xiangya Road, Changsha 410008, China

chronic corticosteroid or other immunosuppressive therapies. However, most patients with CM are immunocompetent in china. Some reports revealed that $10-40 \%$ of HIV-negative patients with CM have no apparent immune deficiency [7-9]. But most studies about prognostic factors have largely focused on the immunocompromised population, so that clinical characters and prognostic factors of immunocompetent patients are not well analyzed.

We sought to conduct a retrospective study to characterize clinical features, laboratory findings, imaging findings and determine potential prognostic factors which predict the outcomes in immunocompetent patients with CM.

(c) The Author(s). 2020 Open Access This article is distributed under the terms of the Creative Commons Attribution 4.0 International License (http://creativecommons.org/licenses/by/4.0/), which permits unrestricted use, distribution, and 


\section{Methods}

We retrospectively reviewed 116 patients with CM from January 2003 to June 2019 in the Department of Neurology, Xiangya Hospital, Central South University, China.

The diagnostic criteria for cryptococcal meningitis was based on the clinical features and findings of cerebrospinal fluid (CSF) after lumbar puncture. Besides, TSPOT, tuberculosis ELISA, AFB stain, culture in CSF were performed to rule out the possibility of tuberculosis.

As we all know, the following situations that are correlated with immunodeficiency will be excluded: History of autoimmune disorders, long-term glucocorticoids or other immunosuppressive therapies, patients with idiopathic CD4 T-cell lymphopenia, HIV infection, malignant tumor, hepatic cirrhosis, end-stage renal failure or diabetes. So that patients without these situations will be regarded as immunocompetent.

In this study, we collected demographic data, major symptoms and signs, findings of routine neuroimaging and laboratory findings. The MRI scan, performed on a 1.5 T MRI scanner (Signa GE, Milwaukee, USA), was independently reviewed by radiologists.

At discharge, the clinical outcome was graded by the Glasgow outcome scale (GOS). Score of 1-4, which indicates death, vegetative status, severe and moderate disability, was considered "unfavorable" clinical outcomes. Score of 5 , which indicates mild or no disability was considered "good" outcomes. Informed consent was obtained from patients or their guardians.

All statistical analyses were performed using the Statistical Package for IBM SPSS Statistics for Windows (version22.0, Chicago, IL, USA). The demographic and clinical data between the good and poor outcome groups were compared. The quantitative variables were compared using two-sample t-test for parametric data and Mann Whitney $U$ test for non-parametric data. The qualitative variables were compared using Pearson's $\chi^{2}$ or Fisher exact test, as appropriate. Finally, potentially prognostic factors for predicting the clinical outcome of $\mathrm{CM}$ were identified using a multiple logistic regression model. P-value $<0.05$ was considered statistically significant.

\section{Results}

Overall, a total of 76 apparently immunocompetent patients with CM were included in this study. The median onset age of patients was 50 (range 16, 77) years old. Headache, fever, vomiting were the three most common symptoms. Demographics and clinical features were shown in Table 1.The median white blood cell (WBC) count in the blood was 8.4 (interquartile range 6.6, $11.8) \times 10^{9} / \mathrm{L}$. $85.5 \%(65 / 76)$ of the patients had abnormal
Table 1 Demographic and clinical profile of apparently immunocompetent patients with Cryptococcal meningitis

\begin{tabular}{ll}
\hline Variable & Value \\
\hline Gender, M/F & $50 / 26(66 \% / 34 \%)$ \\
Age at onset (years) & $50.0(40-62)$ \\
interval from onset to antifungal treatment (day) & $29(15,40)$ \\
duration of antifungal treatment (day) & $31(12.5,54.5)$ \\
Am B administration & $69(90.8 \%)$ \\
Shunt surgery & $11(14.5 \%)$ \\
Main symptoms and signs & \\
Headache & $71(93.4 \%)$ \\
Fever & $48(63.2 \%)$ \\
Vomiting & $38(50.0 \%)$ \\
Impaired consciousness & $15(19.7 \%)$ \\
Visual disturbance & $15(19.7 \%)$ \\
Seizures & $9(11.8 \%)$ \\
Limb weakness & $7(9.2 \%)$ \\
Altered mentation & $7(9.2 \%)$ \\
Mearing impairment & $2(2.6 \%)$ \\
\hline Meningeal irritation positive & $45(59.2 \%)$ \\
\hline
\end{tabular}

Data are $\mathrm{n}$ (\%) or median (interquartile range); $A m B$ Amphotericin $\mathrm{B}, W B C$ White blood cell, CSF Cerebrospinal fluid

CSF opening pressure $(>180 \mathrm{mmH} 2 \mathrm{O})$, in which $51.3 \%(39 / 76)$ of patients were higher than $300 \mathrm{~mm} \mathrm{H} 2 \mathrm{O}$. The median WBC count in the CSF was 50(IQR 18.5, 122.5) $10^{6} / \mathrm{L}$, The median CSF glucose concentration was $1.66(\mathrm{IQR} \quad 0.80,2.85) \mathrm{mmol} / \mathrm{L}$. The median CSF chloride concentration was 117.0 (IQR 113.7, 120.3) $\mathrm{mmol} / \mathrm{L}$. The median CSF protein concentration was 0.85 (IQR 0.54, 1.57) g/L. The sensitivity of the CSF India ink test and culture in our study were 86.8 and $8.7 \%$, respectively. $34.2 \%(26 / 76)$ of patients shown positive india ink test and culture. Laboratory data are presented in Table 2.

MR imaging findings were summarized in Table 2 and Fig. 1. A total of $81.6 \%(62 / 76)$ patients had abnormal image findings. Parenchymal lesions, dilated VirchowRobin spaces and meningeal enhancement were the three most common findings.

At discharge, we further assessed the outcome for all patients by using GOS, 35 patients $(46.1 \%)$ obtained a good outcome.

In a univariate analysis comparing the good outcome group with the unfavorable outcome group, nonparametric test confirmed that unfavorable outcome was associated with lower glucose level of $\operatorname{CSF}(P=0.001)$, and Pearson's $X^{2}$ analysis confirmed that unfavorable outcome was associated with opening pressure of CSF(>300mmH20, $P=0.038)$, impaired consciousness $(P=0.001)$, hydrocephalus $(P=0.045)$, and Shunt surgery 
Table 2 laboratorial findings and Neuroimaging of apparently immunocompetent patients with Cryptococcal meningitis

\begin{tabular}{ll}
\hline Variable & Value \\
\hline Blood WBC count $\left(10^{9} / \mathrm{L}\right)$ & $8.4(6.6,11.8)$ \\
CSF & \\
Opening pressure $(>180 \mathrm{mmH} 2 \mathrm{O})$ & $65(85.5 \%)$ \\
WBC count $\left(10^{6} / \mathrm{L}\right)$ & $50(18.5,122.5)$ \\
Elevated WBC counts & $69(90.8 \%)$ \\
Glucose (mmol/L) & $1.66(0.80,2.85)$ \\
Increased glucose level & $56(73.7 \%)$ \\
Chloride (mmol/L) & $117.0(113.7,120.3)$ \\
Increased chloride level & $55(72.4 \%)$ \\
Protein (g/L) & $0.85(0.54,1.57)$ \\
Increased protein level & $62(81.6 \%)$ \\
India ink test positive & $66(86.8 \%)$ \\
Culture positive & $37(48.7 \%)$ \\
Neuroimaging & \\
Dilated Virchow-Robin spaces & $50(65.8 \%)$ \\
Parenchymal lesions & $41(53.9 \%)$ \\
meningeal enhancement & $16(21.1 \%)$ \\
Hydrocephalus & $11(14.5 \%)$ \\
Gelatinous pseudocyst & $7(9.2 \%)$ \\
\hline
\end{tabular}

Data are $\mathrm{n}$ (\%) or median (interquartile range); $A m B$ Amphotericin B, WBC White blood cell, CSF Cerebrospinal fluid
$(P=0.045)$. Tables 3 and 4 summarize the results of further analyses comparing the quantitative and qualitative variables between the good outcome group and the unfavorable outcome group. Multiple logistic regression analysis confirmed that impaired consciousness and glucose concentration of CSF were prognostic factors which predict the outcome in immunocompetent patients with CM (Table 5).

\section{Discussion}

Environmental resource plays an important role in epidemiology of Cryptococcus neoformans. Previous studies have discovered that cryptococcosis may developed in people after exposure to birds or bird guano $[10,11]$. In the study conducted by XX et al., 20 samples of pigeon droppings $(5 \%, N=400)$ were positive for $C$. neoformans, and pigeon excreta is determined as a favorable environment for growth of this organism [3]. Besides, Pigeon are popular as pet and are considered as a bird of peace in general population; therefore, they raise and care in close with human living area, which contribute to spread of pathogen Cryptococcus neoformans.

$\mathrm{CM}$ has often been described as an opportunistic infection in immunocompromised individuals [12, 13]. Yuchong C, et al. collected 7315 cases with CM and discovered that most common underlying diseases were HIV infection, liver disease, systemic lupus erythematosus, and diabetes mellitus, which are correlated with immunodeficiency, only $17 \%$ of patients had no underlying diseases [14]. That is why most previous studies have

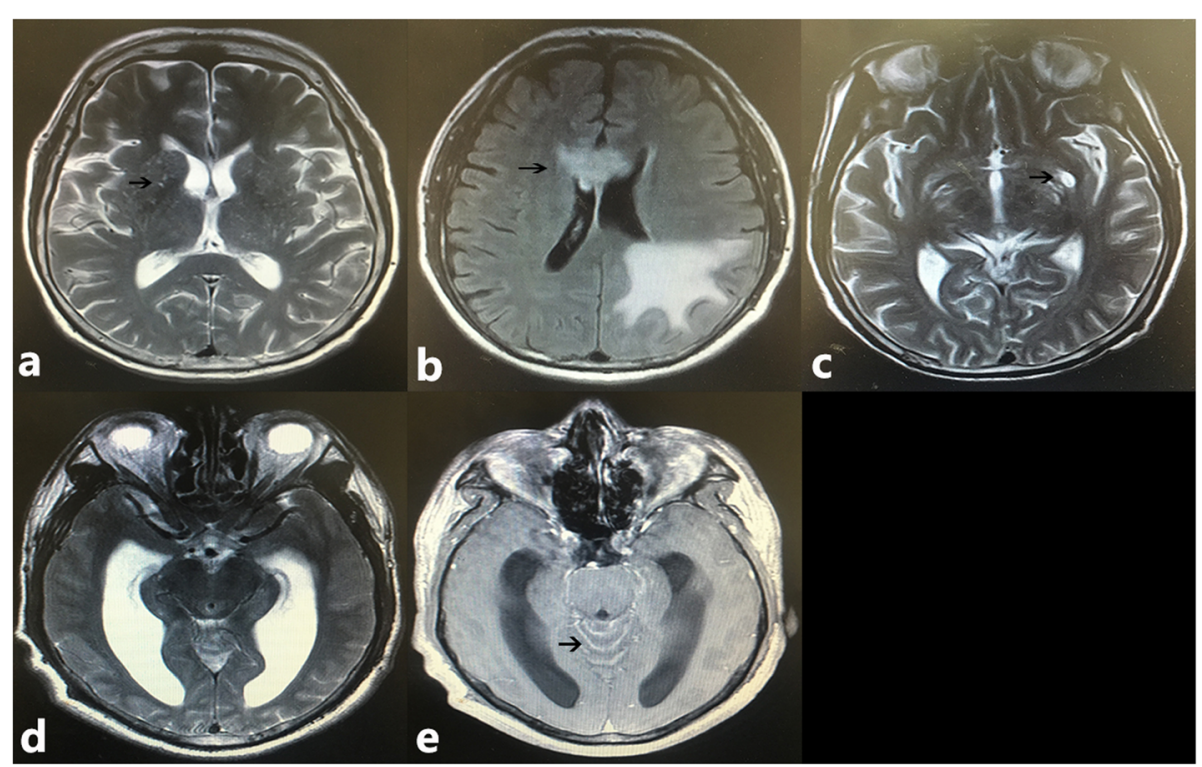

Fig. 1 Neuroimaging characters of patients with cryptococcal meningitis. a T2-W image shows multiple dilated Virchow-Robin spaces (black arrow) in basal ganglia; b Abnormality (black arrow) on FLAIR image within the occipital lobe and corpus callosum; c T2-W image shows a gelatinous pseudocyst (black arrow) in basal ganglia; d hydrocephalus on T2-W image; e Contrast-enhanced image shows meningeal enhancement (black arrow) in cerebellum 
Table 3 Results of univariate analysis identifying variables that differed significantly between the good and unfavorable outcome groups

\begin{tabular}{llll}
\hline Quantitative variable & Good $(n=35)$ & Unfavorable $(n=41)$ & $P$-value \\
\hline T-test & & $52.24 \pm 15.20$ & 0.052 \\
Age at onset (years) & $45.63 \pm 13.80$ & $328.05 \pm 119.80$ & \\
CSF opening pressure $(\mathrm{mmH} 2 \mathrm{O})$ & $290.46 \pm 108.24$ & & \\
Rank sum test & & $38.69 \pm 38.42$ & 0.158 \\
Interval from onset to antifungal treatment (day) & $30.27 \pm 25.65$ & $32.81 \pm 26.43$ & 0.223 \\
Duration of antifungal treatment (day) & $41.61 \pm 36.85$ & $10.04 \pm 3.85$ & 0.346 \\
Blood WBC count $\left(10^{9} / \mathrm{L}\right)$ & $8.96 \pm 4.06$ & $77.39 \pm 104.71$ \\
CSF WBC count(10 $/ \mathrm{L})$ & $92.71 \pm 80.54$ & $1.54 \pm 1.32$ & 0.177 \\
CSF Glucose $(\mathrm{mmol} / \mathrm{L})$ & $2.43 \pm 1.22$ & $115.81 \pm 9.70$ & 0.104 \\
CSF Chloride $(\mathrm{mmol} / \mathrm{L})$ & $117.34 \pm 4.82$ & $1.25 \pm 0.93$ \\
CSF Protein $(\mathrm{g} / \mathrm{L})$ & $0.96 \pm 0.52$ & 0.794 \\
\hline
\end{tabular}

Quantitative results are expressed as the mean \pm standard deviation. CSF Cerebrospinal fluid

focused on individuals with or without AIDS. In fact, $\mathrm{CM}$ has been seen more frequently in immunocompetent individuals in china. The researches from Hongkong and Taiwan show $55-67 \%$ of patients are apparently immunocompetent $[9,15,16]$, and three studies from mainland china demonstrated a high proportion of immunocompetent patients [17-19]. Consistently, we collected 116 patients identified with CM in the present study, and a high proportion of apparently immunocompetent patients (65.5\%) were included. This phenomenon may be explained by the low incidence of AIDS and the lack of full development of organ transplantation in China. Performing Shunt placement reflects the existence of uncontrollable intracranial hypertension that is directly associated with poor clinical manifestation and early death [20-22].

Reviewing the clinical manifestations of the 76 patients in the present study, headache $(71 / 76,93.4 \%)$, fever (71/

Table 4 Analysis identifying qualitative variables that differed significantly between the good and unfavorable outcome groups

\begin{tabular}{|c|c|c|c|}
\hline Qualitative variables & Good $(n=35)$ & Unfavorable $(n=41)$ & $P$-value \\
\hline Gender (male) & 23 & 27 & 0.990 \\
\hline Am $B$ administration & 33 & 36 & 0.565 \\
\hline Shunt surgery & 2 & 9 & 0.045 \\
\hline Headache & 34 & 37 & 0.456 \\
\hline Fever & 23 & 25 & 0.669 \\
\hline Vomiting & 19 & 19 & 0.490 \\
\hline Impaired consciousness & 1 & 14 & 0.001 \\
\hline Visual disturbance & 5 & 10 & 0.270 \\
\hline Seizures & 1 & 8 & 0.065 \\
\hline Limb weakness & 3 & 4 & 1.000 \\
\hline Altered mentation & 2 & 5 & 0.565 \\
\hline Hearing impairment & 1 & 1 & 0.910 \\
\hline CSF Opening pressure $(>300 \mathrm{mmH} 2 \mathrm{O})$ & 13 & 25 & 0.038 \\
\hline Meningeal irritation positive & 17 & 28 & 0.081 \\
\hline India ink test and culture positive & 15 & 11 & 0.142 \\
\hline Dilated VRS & 23 & 27 & 0.990 \\
\hline Parenchymal lesions & 19 & 22 & 0.956 \\
\hline Meningeal enhancement & 6 & 10 & 0.440 \\
\hline Hydrocephalus & 2 & 9 & 0.045 \\
\hline Gelatinous pseudocyst & 3 & 4 & 1.000 \\
\hline
\end{tabular}


Table 5 Results of backward stepwise multiple logistic regression analysis of variables that differed significantly between the good and unfavorable outcome groups

\begin{tabular}{lll}
\hline Variable & \multicolumn{2}{l}{ Engel classification } \\
\cline { 2 - 3 } & Regression coefficient & $P$-value \\
\hline CSF Glucose & -0.575 & 0.012 \\
Impaired consciousness & 2.683 & 0.015 \\
Constant & 0.779 & 0.130 \\
\hline
\end{tabular}

CSF Cerebrospinal fluid

$76,93.4 \%)$, vomiting $(38 / 76,50 \%)$ were common but not significantly different between the good outcome group and unfavorable groups, almost consistent with previous studies [5, 9].. Several studies focused on non-HIV patients reported the correlation between initial consciousness level and therapeutic outcome [19, 23, 24]. Consistently, in the present study, logistic regression analysis demonstrated that CM patients with impaired consciousness had a significantly higher probability of an unfavorable outcome.

Several previous studies confirm that high CSF opening pressure is prognostic factors independently associated with unfavorable outcome or increased odds of mortality [5, 25], in present study, CSF opening pressur$\mathrm{e}(>300 \mathrm{mmH} 2 \mathrm{O})$ is associated with unfavorable outcome, but our multivariate logistic regression analyses did not identify it as a significant prognostic factor for outcome which may due to small sample size. CSF opening pressure requires further analysis in larger cohorts of immunocompetent patients. Decreased CSF glucose concentration is used as indicators in the process of diagnosing community-acquired meningitis [26]. In previous studies from china, patients with Decreased CSF glucose level tended to have worse outcome [18, 27], and this also occurred in our patient population. Infection with Cryptococcus neoformans caused dysfunction of the blood-brain barrier and presentation of cryptococcus in CSF [28], which result in the abnormality of glucose transportation and increased glycolysis, respectively [29]. Those factors lead to decreased CSF glucose level in CM patients.

In a study focused on organ transplant recipients with CM, only sixteen patients $(29 \% / N=55)$ had abnormal findings [30]. In contrast, more immunocompetent patients $(81.6 \% / N=76)$ show abnormal MRI findings in our patient population. An autopsy research compared immunocompetent and immunocompromised patients found that lesions were more restricted to the perivascular space [31], and VRS are perivascular spaces at thalamus, basal ganglia, periventricular white matter and the cerebellum [32]. Consistently, Dilated VRS is the most common finding of neuroimaging in our study. But the former research maybe underestimates the presence of parenchymal lesion because of small case size $(N=27)$. In fact, up to 41 patients (53.9\%) show parenchymal lesion in our cases, which is in accordance with our previous study focused on neuroimaging findings $(61.1 \%$ / $N=18$ ) [33]. This may be explained by cryptococcal infection that spread into the parenchyma through the VRS during disease progression.

MRI scan is routine and important examination in early diagnosis of CNS infection, we try to find some imaging characters to predict the outcome of $\mathrm{CM}$ patients. Hydrocephalus is common abnormal finding of imaging, which has been reported in several studies as an important factor for unsatisfactory outcome in HIVnegative patients with CM [34] and tuberculous meningitis [35], and hydrocephalus is significantly associated with unfavorable outcome in the present study. However, it doesn't be identified as a predictor Independent prognostic factor, which may be due to too few cases with hydrocephalus. The present study is limited by following factors. First, our study is a retrospective, and single-center design and small sample size may lead to neglect and underestimation of some significant predictors. Second, variant administration of antifungal treatment made it difficult to investigate their influences on outcome. Third, lack of findings about serotypes and varieties of Cryptococcus neoformans may result in neglect of more correlation between clinical data and microbiological findings. Forth, clinical outcome was graded by the Glasgow outcome scale at discharge without follow-up. So that prospective clinical researches with a large population, multicenter, and long-term follow up are required in order to yield more reliable results.

\section{Conclusion}

Impaired consciousness and decreased glucose concentration of CSF increase the likelihood of unfavorable outcome in CM patients. Prior research and our findings indicates that absences of Hydrocephalus and CSF Opening pressure $(<300 \mathrm{mmH} 2 \mathrm{O})$ are beneficial for achieving a favorable outcome.

\section{Abbreviations \\ CM: Cryptococcal meningitis; CNS: Central nervous system; CSF: Cerebrospinal fluid; GOS: Glasgow outcome scale; HIV: Human Immunodeficiency Virus; VRS: Virchow-Robin spaces}

\section{Acknowledgments}

We thank all our colleagues at the Department of Neurology.

\section{Authors' contributions}

All authors had full access to all the data in the study and take responsibility for the integrity of the data and the accuracy of the data analysis. Conceptualization, CZ and ZT; Methodology, ZT, CZ; Investigation, Formal Analysis, Resources, Visualization, Writing, Original Draft, CZ and ZT, Writing Review \& Editing, Supervision, FT. All authors have read and approved the manuscript. 


\section{Funding}

This study was supported by National key R\&D program of China (2017YFC1310003) and Natural Science Foundation of China (81571276). The funders had no role in the design of the study and collection, analysis, and interpretation of data and in writing the manuscript.

\section{Availability of data and materials}

The data that support the findings of this study are available from the corresponding author via E-mail upon reasonable request.

\section{Ethics approval and consent to participate}

All subjects gave their verbal informed consent prior to their inclusion in the study. Written consent to participate was waived as the present study was a retrospective study using deidentified data, and patients will spend additional time and transportation costs for obtaining written consent to participate after discharge, which will increase the burden on patients. This study was approved by the Research Ethics Committee of the Xiangya Hospital, and was conducted according to the principles expressed in the Declaration of Helsinki.

\section{Consent for publication}

Not applicable.

\section{Competing interests}

The authors declare that they have no competing interests.

Received: 11 September 2019 Accepted: 14 January 2020

Published online: 22 January 2020

\section{References}

1. Hagen F, Lumbsch HT, Arsic Arsenijevic V, et al. Importance of Resolving Fungal Nomenclature: the Case of Multiple Pathogenic Species in the Cryptococcus Genus. mSphere. 2017;2(4). https://doi.org/10.1128/mSphere. 00238-17

2. Badali $\mathrm{H}$, Alian $\mathrm{S}$, Fakhim $\mathrm{H}$, et al. Cryptococcal meningitis due to Cryptococcus neoformans genotype AFLP1/NNI in Iran: a review of the literature. Mycoses. 2015:58(12):689-93.

3. Agha Kuchak Afshari S, Shokohi T, Aghili R, et al. Epidemiology and molecular characterization of Cryptococcus neoformans isolated from pigeon excreta in Mazandaran province, northern Iran. J Mycol Med. 2012; 22(2):160-6.

4. Guo LY, Liu LL, Liu Y, et al. Characteristics and outcomes of cryptococcal meningitis in HIV seronegative children in Beijing, China, 2002-2013. BMC Infect Dis. 2016;16:635.

5. Chen $\mathrm{CH}$, Sy HN, Lin LJ, et al. Epidemiological characterization and prognostic factors in patients with confirmed cerebral cryptococcosis in Central Taiwan. J Venom Anim Toxins Incl Trop Dis. 2015;21:12.

6. Bandalizadeh Z, Shokohi T, Badali H, et al. Molecular epidemiology and antifungal susceptibility profiles of clinical Cryptococcus neoformans/ Cryptococcus gattii species complex. J Med Microbiol. 2019. https://doi.org/ 10.1099/jmm.0.001101.

7. Li M, Chen Z, Xu L, et al. A comparison of the clinical characteristics and outcomes of Cryptococcal meningitis in HIV-negative individuals with and without immunosuppression. Neurologist. 2019;24(1):1-5.

8. Ecevit IZ, Clancy CJ, Schmalfuss IM, et al. The poor prognosis of central nervous system cryptococcosis among nonimmunosuppressed patients: a call for better disease recognition and evaluation of adjuncts to antifungal therapy. Clin Infect Dis. 2006;42:1443-7.

9. Lui G, Lee $\mathrm{N}$, Ip M, et al. Cryptococcosis in apparently immunocompetent patients. QJM. 2006;99:143-51.

10. Lagrou K, van Eldere J, Keuleers S, et al. Zoonotic transmission of Cryptococcus neoformans from a magpie to an immunocompetent patient. J Intern Med. 2005;257:385-8.

11. Nosanchuk JD, Shoham S, Fries BC, et al. Evidence of zoonotic transmission of Cryptococcus neoformans from a pet cockatoo to an immunocompromised patient. Ann Intern Med. 2000;132:205-8.

12. Sonneville R, Magalhaes E, Meyfroidt G. Central nervous system infections in immunocompromised patients. Curr Opin Crit Care. 2017;23(2):128-33.

13. Henao-Martínez AF, Chastain DB, Franco-Paredes C. Treatment of cryptococcosis in non-HIV immunocompromised patients. Curr Opin Infect Dis. 2018;31(4):278-85
14. Yuchong C, Fubin C, Jianghan C, et al. Cryptococcosis in China (1985-2010): review of cases from Chinese database. Mycopathologia. 2012;173(5-6):329 35

15. Lu CH, Chang HW, Chuang YC. The prognostic factors of cryptococcal meningitis in HIV-negative patients. J Hospital Infect. 1999;42:313-20.

16. Shih CC, Chen YC, Chang SC, et al. Cryptococcal meningitis in non-HIVinfected patients. QJM. 2000;93:245-51.

17. Wu MH, Xu ZY, Weng XH, et al. A clinical analysis of 74 cases of cryptococcosis. Acta Academiae Med Shanghai. 1987;14:457-9.

18. Yao Z, Liao W, Chen R. Management of cryptococcosis in non HIV-related patients. Med Mycol. 2005:43:245-51.

19. Zhu LP, Wu JQ, Xu B, et al. Cryptococcal meningitis in non-HIV-infected patients in a Chinese tertiary care hospital, 1997-2007. Med Mycol. 2010;48: 570-9.

20. Saag MS, Graybill RJ, Larsen RA, et al. Practice guidelines for the management of cryptococcal disease. Clin Infect Dis. 2000;30:710-8.

21. Vidal JE, Gerhardt J, Peixoto de Miranda EJ, et al. Role of quantitative CSF microscopy to predict culture status and outcome in HIV-associated cryptococcal meningitis in a Brazilian cohort. Diagn Microbiol Infect Dis. 2012;73:68-73.

22. Liliang $P C$, Liang $C L$, Chang WN, et al. Shunt surgery for hydrocephalus complicating cryptococcal meningitis in human immunodeficiency virusnegative patients. Clin Infect Dis. 2003;37:673-8.

23. Hung $C W$, Chang $W N$, Kung $C T$, et al. Predictors and long-term outcome of seizures in human immuno-deficiency virus (HIV)-negative cryptococcal meningitis. BMC Neurol. 2014;14:208.

24. Saag MS, Powderly WG, Cloud GA, et al. Comparison of amphotericin B with fluconazole in the treatment of acute AIDS-associated cryptococcal meningitis. The NIAID mycoses study group and the AIDS Clinical Trials Group. N Engl J Med. 1992;326(2):83-9.

25. Liu $Y$, Jiang $Y$, Wu A, et al. Prognostic significance of serum antioxidant parameters in immunocompetent patients with cryptococcal meningitis. Eur J Clin Microbiol Infect Dis. 2012;31(9):2359-67.

26. Welch $H$, Hasbun R. Lumbar puncture and cerebrospinal fluid analysis. In: Aminoff M, Boller F, Swaab D, editors. editors Handbook of clinical neurology; 2010. p. 31-49.

27. Cao W, Jian C, Zhang $H$, et al. Comparison of clinical features and prognostic factors of Cryptococcal meningitis caused by Cryptococcus neoformans in patients with and without pulmonary nodules. Mycopathologia. 2019;184(1):73-80.

28. Severien C, Jacobs KH, Schoenemann W. Marked pleocytosis and hypoglycorrhachia in coxsackie meningitis. Pediatr Infect Dis. 1994;13:322-3.

29. Dzupova O, Rozsypal H, Prochazka B, et al. Acute bacterial meningitis in adults: predictors of outcome. Scand J Infect Dis. 2009:41:348-54.

30. Singh N, Lortholary $\mathrm{O}$, Dromer $\mathrm{F}$, et al. Central nervous system cryptococcosis in solid organ transplant recipients: clinical relevance of abnormal neuroimaging findings. Transplantation. 2008;86(5):647-51.

31. Lee SC, Dickson DW, Casadevall A. Pathology of cryptococcal meningoencephalitis: analysis of 27 patients with pathogenetic implications. Hum Pathol. 1996:27:839-47.

32. Kwee RM, Kwee TC. Virchow-Robin spaces at MR imaging. Radiographies. 2007;27:1071-86.

33. Tan $Z R$, Long $X Y$, Li GL, et al. Spectrum of neuroimaging findings in cryptococcal meningitis in immunocompetent patients in China - a series of 18 cases. J Neurol Sci. 2016:368:132-7.

34. Liao CH, Chi CY, Wang YJ, et al. Different presentations and outcomes between HIV-infected and HIV-uninfected patients with Cryptococcal meningitis. J Microbiol Immunol Infect. 2012;45(4):296-304

35. Qu J, Zhou T, Zhong C, et al. Comparison of clinical features and prognostic factors in HIV-negative adults with cryptococcal meningitis and tuberculous meningitis: a retrospective study. BMC Infect Dis. 2017;17(1):51.

\section{Publisher's Note}

Springer Nature remains neutral with regard to jurisdictional claims in published maps and institutional affiliations. 